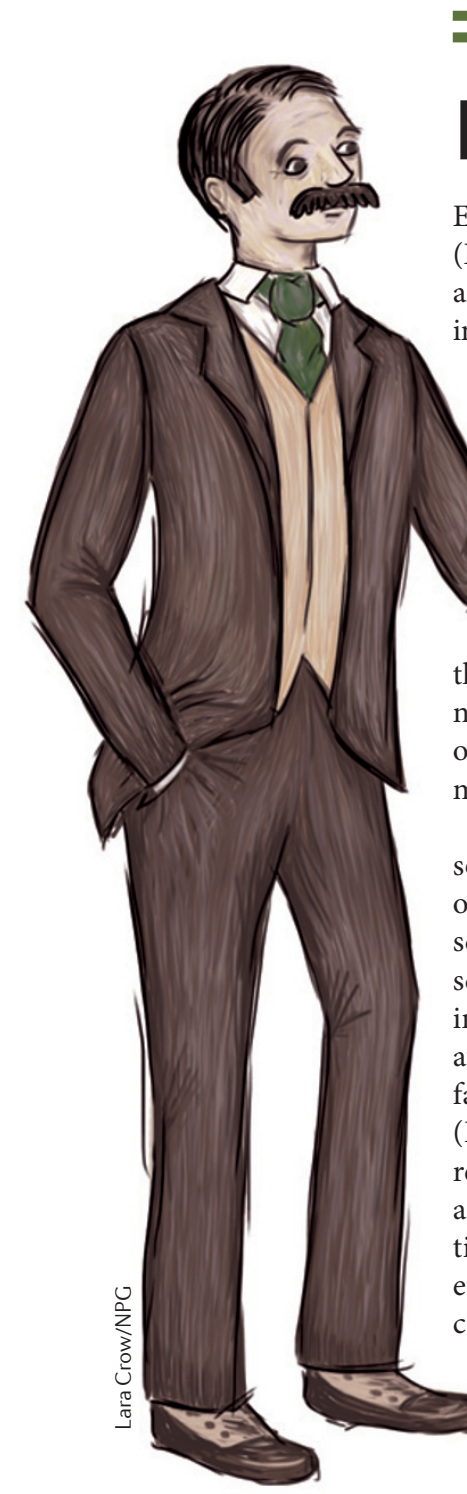

\title{
Epithelial to mesenchymal and back again
}

Epithelial-mesenchymal transition (EMT) is associated with metastasis, and it is thought that EMT confers invasive and stem cell-like features that allow cells to disseminate; whereas, the
reverse process 1. $1 \begin{aligned} & -\mathrm{MET}-\text { is } \\ & \text { thought to }\end{aligned}$ 1) (') allow growth of

these cells at distant sites. Two papers now provide evidence for this idea of plasticity in epithelial and mesenchymal transitions in vivo.

EMT is a developmental process; so, to identify additional regulators of EMT, Ocaña and colleagues screened chick embryos for transcription factors that are involved in early developmental stages. The authors identified the transcription factor paired related homeobox 1 (PRRX1). PRRX1 expression correlated with invasive phenotypes and TWIST1 (an EMT transcription factor) expression in zebrafish embryos and a panel of human cancer cell lines. Furthermore,

knockdown of PRRX1 or TWIST1

decreased the ability of BT-549

human breast carcinoma cells

to degrade collagen, whereas expression of PRRX1 induced the invasion of HBL-100 breast epithelial cells (which are non-invasive and which express TWIST1 but not PRRX1).

Is PRRX1 involved in metastasis? Cells expressing PRRX1 that EMT reversion were injected into the tail veins of is crucial for metastatic outgrowth either PRRX1 alone or both PRRX1 and TWIST1 were knocked down developed primary tumours, whereas implantation of BT-549 cells expressing PRRX1 did not. The tumours that formed did not exhibit an invasive margin and did not form overt metastases. Further experiments revealed that PRRX1 loss induces MET (in cells that have undergone EMT), stem cell-like properties and proliferation. Additionally, analyses of gene expression data sets revealed that high levels of $P R R X 1$ mRNA correlated with relapse-free survival in breast adenocarcinoma and lung squamous cell carcinoma. Together, these data show that PRRX1 expression is associated with EMT and invasion, but its expression must be lost at later stages for metastatic colonization.

To investigate the plasticity of epithelial and mesenchymal transitions, Tsai and colleagues analysed the expression of TWIST1 in primary human breast carcinoma and matched lymph node metastases. Of the primary tumours with high TWIST1 expression, most of the matched lymph node metastases no longer expressed TWIST1. The authors generated transgenic mice in which Twist 1 expression could be induced in the skin by doxycycline and used the DMBA-TPA carcinogenesis protocol to induce papillomas. Doxycycline was then applied orally (to induce Twist 1 in all skin tumour cells even if they had disseminated) or topically (to induce Twist 1 at primary sites), and both methods increased the conversion rate to invasive squamous cell carcinoma compared with controls. However, topical induction of Twist 1 significantly increased the metastasis frequency compared with mice receiving oral doxycycline. This indicates that turning off Twist 1 expression (which occurs as cells disseminate from the primary sites) is required for metastasis formation. Consistently, the metastases that formed in mice treated with oral or topical doxycycline no longer expressed TWIST1 and had epithelial characteristics, indicating EMT reversion.

At what stage is EMT reversed in metastasis? Analyses of peripheral blood from these mice revealed that Twist 1 induction doubled the number of circulating tumour cells, and these cells expressed TWIST1. Tail-vein injection of primary tumour cells expressing TWIST1 also extravasated from the lung vasculature. Importantly, turning off Twist1 expression in these cells allowed their proliferation in vivo. This indicates that EMT reversion is crucial for metastatic outgrowth.

These two papers provide in vivo evidence for the plasticity of epithelial and mesenchymal transitions, indicating that EMT (induced by PRRX1 and/or TWIST1) is required for invasion, intravasation and extravasation, and that the suppression of EMT regulators (and consequently EMT reversion) is required for metastatic outgrowth.

Gemma K. Alderton

ORIGINAL RESEARCH PAPERS Ocaña, O. H.

et al. Metastatic colonization requires the repression of the epithelial-mesenchymal transition inducer Prrx1. Cancer Cell 11 Dec 2012 (doi:10.1016/j.ccr.2012.10.012) | Tsai, J. H. et al. Spatiotemporal regulation of epithelialmesenchymal transition is essential for squamous cell carcinoma metastasis. Cancer Cell 11 Dec 2012 (doi:10.1016/j. ccr.2012.09.022) 